\title{
TANNIC ACID-IMMOBILIZED CELLULOSE NANOFIBER PREPARED BY ESTERIFICATION USING POLYCARBOXYLIC ACID
}

\author{
AKIO KUMAGAI and TAKASHI ENDO \\ Research Institute for Sustainable Chemistry, Department of Materials and Chemistry, \\ National Institute of Advanced Industrial Science and Technology (AIST), 3-11-32 Kagamiyama, \\ Higashi-Hiroshima, Hiroshima 737-0046, Japan \\ Corresponding author: A. Kumagai,a-kumagai@aist.go.jp
}

Received April 10, 2020

Tannic acid (TA) exhibits unique adsorption capacity, while cellulose nanofiber (CNF) has excellent properties as immobilization carrier. In this study, to combine the excellent properties of both TA and CNF, tannic acid-immobilized cellulose nanofiber (TA-CNF) was prepared by esterification, using butane-1,2,3,4-tetracarboxylic acid (BTCA). The simple esterification method achieved immobilization of TA on cellulose nanofibrils, which was verified by attenuated total reflection Fourier transform infrared spectroscopy. The average fiber diameter of TA-CNF was $4.7 \mathrm{~nm}$, as determined by atomic force microscopy characterization. The protein adsorption capacities of TA-CNF and TAadsorbed CNF were compared, suggesting that the immobilization of TA on CNF is important for manifesting the protein adsorption capacity of TA. Since TA can adsorb various materials, besides proteins, TA-CNF may have the potential to expand the application of $\mathrm{CNF}$ as an immobilization carrier.

Keywords: cellulose nanofiber, tannic acid, immobilization, adsorption, quartz crystal microbalance

\section{INTRODUCTION}

Cellulose is the most abundant biopolymer on earth and has attracted significant attention for nanoscale applications. ${ }^{1}$ The naturally derived cellulose nanomaterial, i.e., cellulose nanofiber (CNF), possesses typical nanomaterial properties, such as a high specific surface area, high mechanical durability, and high reactivity. Therefore, CNF can act as a carrier for the immobilization of proteins, metallic nanoparticles, drugs, etc. $^{2-4}$

Tannic acid (TA) is a water-soluble polyphenol, comprising gallic acid glucoside, which can be easily extracted from various plants. $^{5}$ TA has been applied to protein immobilization, metal removal, drug delivery, and cytoprotection, due to its unique adsorption ability, owing to its di-galloyl ester groups, low price, and nontoxicity. ${ }^{6-9}$ However, for all these applications, water-soluble TA should be immobilized on a water-insoluble support. ${ }^{10,11}$ The preparation of TA-immobilized cellulose materials using chemically modified celluloses, such as cellulose acetate and alkali-treated cellulose, have been reported previously. ${ }^{12-14}$ The immobilization of TA on chemically modified cellulose has been investigated by several methods, such as crosslinking using epichlorohydrin as crosslinking agent and hydrogen bonding with poly(ethylene glycol). ${ }^{12-14}$

Herein, we conducted the immobilization of TA on chemically unmodified cellulose via esterification using polycarboxylic acid. Butane1,2,3,4-tetracarboxylic acid (BTCA), a polyvalent carboxylic acid, has been extensively investigated as a crosslinking agent to introduce anti-wrinkle properties to cotton fabrics. ${ }^{15,16}$ The crosslinks are formed by esterification between the hydroxyl groups of cellulose and carboxyl groups of BTCA. If TA is also present in the same reaction system, it will form crosslinks with BTCA as well and will be immobilized onto the cellulose (Fig. 1a).

Further, crosslinking using BTCA introduces carboxyl groups into the cellulose (Fig. 1a). The introduction of a negatively charged substrate enables the preparation of fine CNFs, with widths in the order of several nanometers. Therefore, the methods used to prepare fine CNFs containing 
carboxyl groups, phosphate groups, and sulfate groups were studied. ${ }^{17-19}$ If carboxyl groups are introduced by BTCA, the electrostatic repulsion between the carboxyl groups enables the nanofibrillation of the cellulose materials.

Hydrogels and aerogels prepared by the direct adsorption of TA onto CNF and crosslinking it with other polymers, such as polyvinyl alcoholborax and cardanol-derived siloxane, have been reported in several studies. ${ }^{20,21}$ However, in this study, both the immobilization of TA and nanofibrillation were achieved using BTCA as crosslinking agent. The immobilization of TA on CNF was confirmed by attenuated total reflection Fourier transform infrared spectroscopy (ATRFTIR), and nanofibrillation was evaluated by microscopic observations. In addition, to evaluate the ability of the material to act as a support, the protein adsorption capacity of the TAimmobilized CNF (TA-CNF) was studied as a function of the adsorption abilities of TA by performing a quartz crystal microbalance (QCM) analysis to evaluate the adsorption capacity.

\section{EXPERIMENTAL}

Materials

Commercial cellulose powder (KC Flock W-400G, Nippon Paper Industries Co., Ltd., Japan), with an average particle size of $24 \mu \mathrm{m}$, was used as the starting material for preparing CNF. TA, BTCA, sodium hypophosphite (SHP), sodium hydroxide $(\mathrm{NaOH})$ and other chemicals were of analytical grade and thus used without further purification, after being purchased from commercial sources. BTCA and SHP were used as esterification agent and catalyst, respectively.

\section{Preparation of TA-CNF}

An aqueous solution of TA, BTCA and SHP was prepared at room temperature by stirring, using a magnetic stirrer. Cellulose powder $(1 \quad \mathrm{wt} \%)$ was dispersed in the prepared solution, at a weight ratio similar to that of TA (CNF/TA/BTCA/SHP = 1:1:1:0.5), and stirred for $1 \mathrm{~h}$. The cellulose powder suspension was filtered through a polytetrafluoroethylene membrane filter (H010A090C, pore size: $0.1 \mu \mathrm{m}$, Advantec Toyo Co., Ltd., Japan) under suction for $15 \mathrm{~min}$. The separated cellulose powder was dried in a constant-temperature drying oven (ONW-300S, AzOne Co., Japan) at $80{ }^{\circ} \mathrm{C}$ for 10 min, and then cured at $160{ }^{\circ} \mathrm{C}$ for $10 \mathrm{~min}$ in the oven to facilitate the esterification with TA. Then, the cured cellulose powder was dispersed in distilled water and washed via centrifugation three times at room temperature. Sodium hydroxide $(\mathrm{NaOH})$ solution $(0.1$ $\mathrm{N})$ was added to the washed cellulose powder suspension until its $\mathrm{pH}$ reached 10 . Subsequently, the excess $\mathrm{NaOH}$ was washed away with distilled water by suction filtration. The modified cellulose powder dispersed in distilled water was adjusted to $1 \mathrm{wt} \%$. Then, fibrillation of the corresponding $1 \%$ modified cellulose powder solution was performed using a highpressure homogenizer (Masscomizer MMX-L100, Masuko Sangyo Co., Ltd., Japan) at a pressure of $~ 200$ $\mathrm{MPa}$, five times. Unmodified CNF was prepared by high-pressure homogenization under similar conditions using commercial cellulose powder. Because of the difficulties in its direct homogenization, the cellulose powder was first fibrillated by a disk mill, according to a method reported in another study. ${ }^{22}$ To prepare the TA-adsorbed CNF, unmodified CNF was dispersed in water at a CNF/TA weight ratio of $1: 1$, stirred for $1 \mathrm{~h}$, and then washed with distilled water by suction filtration.

\section{Characterization}

The morphologies of the prepared CNF were observed using field-emission scanning electron microscopy (FE-SEM) (S-4800, Hitachi HighTechnologies Co., Japan) at $1 \mathrm{kV}$. The sample for FESEM was freeze-dried with tert-butyl alcohol substitution and fixed with osmium tetroxide. Atomic force microscopy (AFM) (JSPM-5200, JEOL Co., Ltd., Japan) in tapping mode, using aluminum reflex-coated silicon cantilevers (PPP-NCHR, NanoWorld AG, Switzerland), was used to evaluate the fiber diameter. ATR-FTIR spectroscopy was performed using a FT-IR spectrometer (Spectrum GX, PerkinElmer Co., Ltd., USA) to confirm the immobilization of TA on CNF by esterification via BTCA.

\section{Evaluation of protein adsorption capacity}

QCM apparatus (Q-Sense E1, Biolin Scientific AB, Sweden) was used for investigating the protein adsorption to TA-CNF. A TA-CNF-coated QCM gold sensor (QSX 301, Biolin Scientific AB, Sweden) was prepared using a centrifuged supernatant of $0.1 \mathrm{wt} \%$ TA-CNF suspension, according to the previously described procedure. ${ }^{22}$

The TA-CNF-coated sensor was assembled in the QCM flow chamber, and Milli-Q water was pumped through the chamber. Once the frequency profile of the sensor reached stability, $0.05 \mathrm{mg} / \mathrm{mL}$ of bovine serum albumin (BSA) was introduced and adsorbed for 30 min. Thereafter, Milli-Q water was introduced for 20 min to wash the non-specifically adsorbed BSA. The temperature was set to $25{ }^{\circ} \mathrm{C}$ and the flow rate to 50 $\mu \mathrm{L} / \mathrm{min}$ in all the cases. QCM analyses were also conducted using an unmodified CNF-coated sensor and TA-adsorbed CNF-coated sensor.

\section{RESULTS AND DISCUSSION}

This study set out to prepare TA-CNF to expand the application of CNF as an immobilization carrier. The proposed reaction for 
preparing TA-CNF using BTCA as an esterification agent is shown in Figure 1a. The carboxyl groups in BTCA form ester bonds with the hydroxyl groups on cellulose or TA. Since BTCA has multiple carboxyl groups, it can form ester bonds with two or more cellulose or TA molecules, leading to the crosslinking of celluloses and the immobilization of TA on CNF. The immobilization of TA on CNF was confirmed by ATR-FTIR (Fig. 1b and 1c).

The ATR-FTIR spectra for the unmodified $\mathrm{CNF}$ and TA-adsorbed $\mathrm{CNF}$ were compared to that of TA-CNF. The main difference between the unmodified $\mathrm{CNF}$ and TA-CNF was the appearance of a peak at $1716 \mathrm{~cm}^{-1}$, which can be attributed to the carbonyl band of the TA ester and the carboxyl carbonyl band of BTCA. The peak at $1610 \mathrm{~cm}^{-1}$, which can be attributed to the $\mathrm{C}=\mathrm{C}$ stretching vibration of the TA aromatic ring, was also observed in the spectrum of TA-CNF. These peaks were also observed in the spectrum of TAadsorbed CNF, but disappeared after the $\mathrm{NaOH}$ treatment performed during the preparation of TA-CNF (Fig. 1c). However, for TA-CNF, the peak corresponding to the carbonyl band (1716 $\mathrm{cm}^{-1}$ ) remained, and the peak at $1580 \mathrm{~cm}^{-1}$, which can be attributed to the carboxylate carbonyl band of BTCA, was observed (Fig. 1c). When TA-CNF is treated with the solution of higher $\mathrm{pH}(\mathrm{NaOH})$, the free carboxyl groups of TA-CNF are converted to carboxylate anions. Therefore, the ATR-FTIR spectra confirmed the immobilization of TA on CNF by esterification with BTCA.

Figure 2 shows the morphologies of the three differently prepared CNFs, as observed by FESEM. There was no significant change in the morphology between the unmodified CNF and TA-adsorbed CNF (Fig. 2a and 2b). In contrast, TA-CNF had a finer and looser structure than those of the unmodified CNF and TA-adsorbed CNF (Fig. 2c). In addition, the fiber diameters estimated by AFM were of $4.7 \pm 1.4 \mathrm{~nm}$ for TACNF (Fig. 2f) and $14.8 \pm 2.9 \mathrm{~nm}$ for unmodified $\mathrm{CNF}$ and TA-adsorbed CNF (Fig. 2d and 2e). The diameter of TA-CNF was close to that of CNF prepared by another chemical treatment, such as 2,2,6,6-tetramethylpiperidine-1-oxyl (TEMPO)oxidation. ${ }^{17}$ That is, esterification via BTCA achieved not only the immobilization of TA, but also the nanofibrillation of cellulose materials.
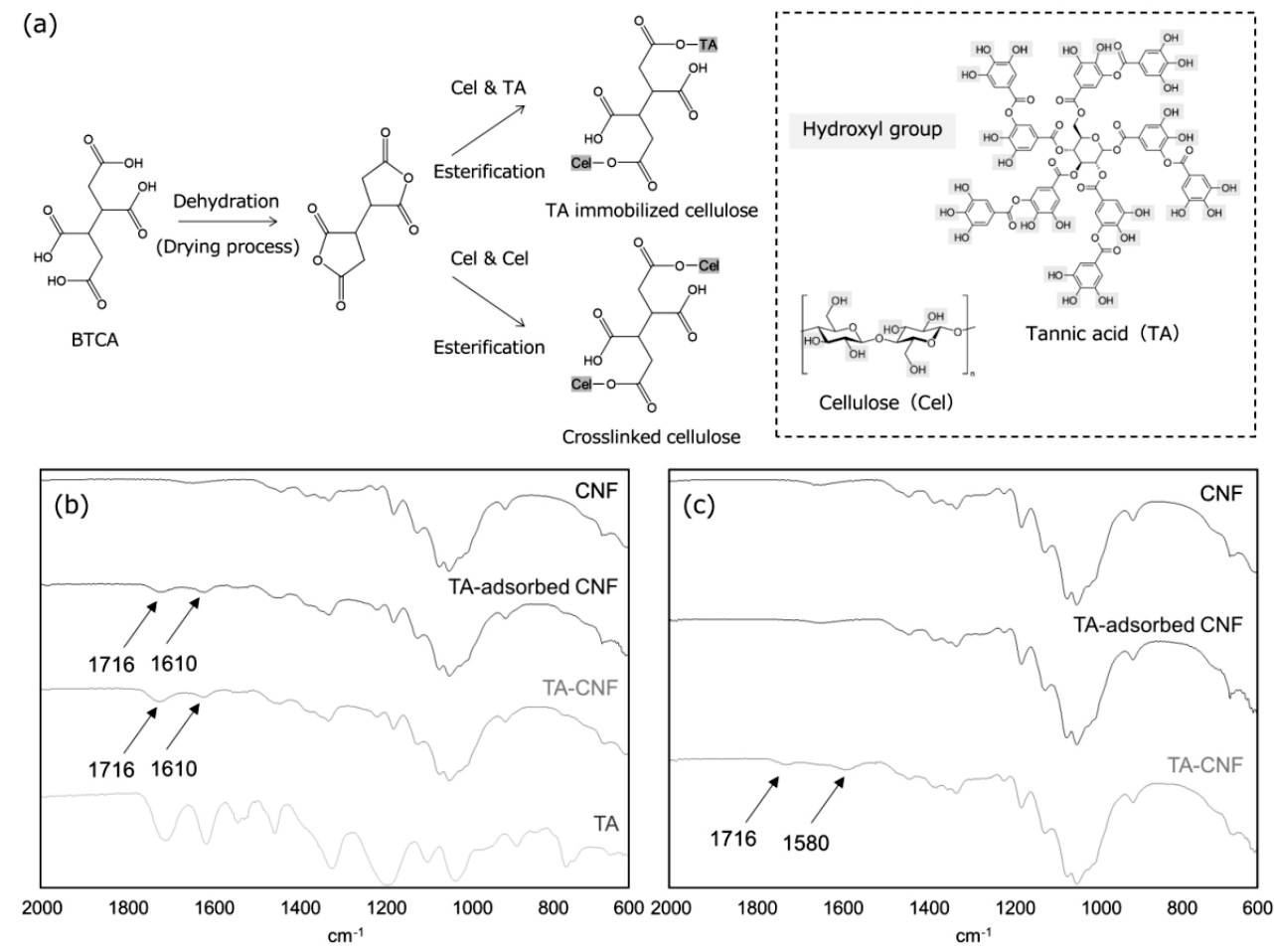

Figure 1: Reaction between TA and cellulose using BTCA as esterification agent (a), ATR-FTIR spectra of TA, TAadsorbed CNF, TA-CNF, and CNF before (b) and after (c) sodium hydroxide treatments 

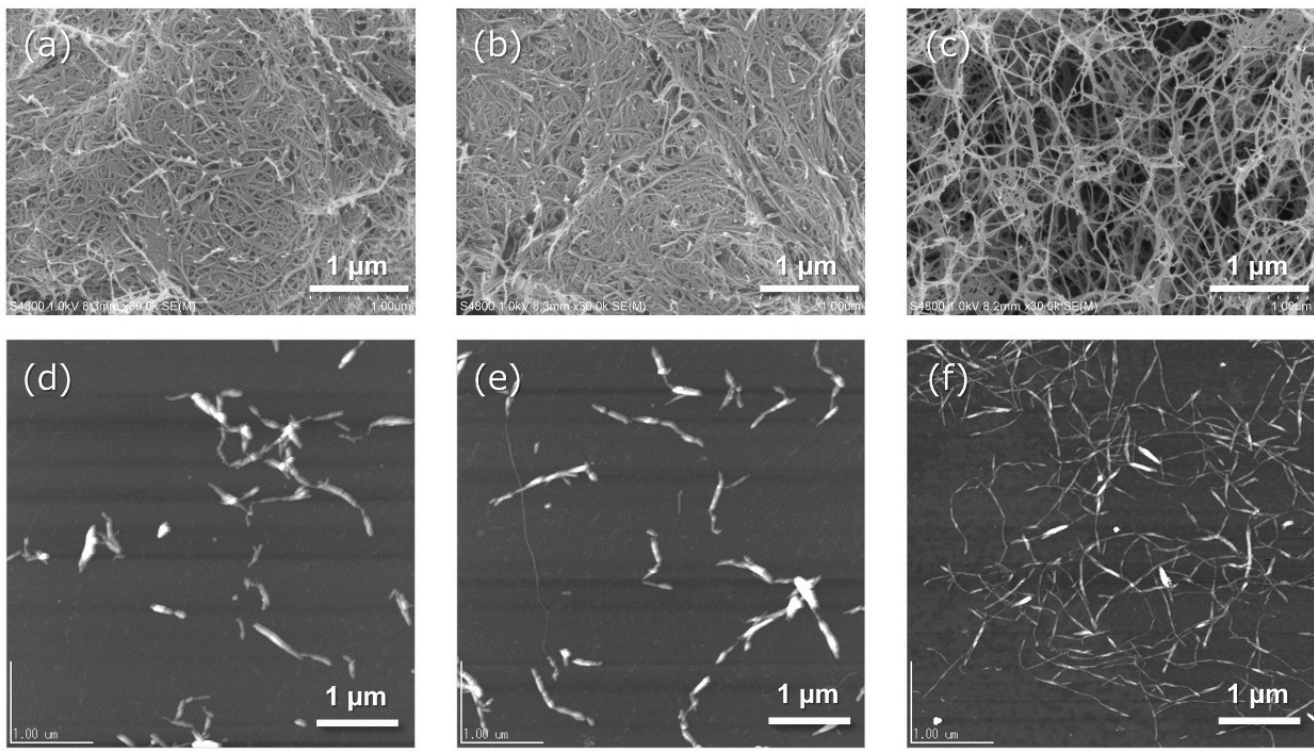

Figure 2: FE-SEM images of freeze-dried CNF (a-c), AFM height images of CNF (d-f); CNF ( $a$ and d), TA-adsorbed CNF (b and e), TA-CNF (c and f)

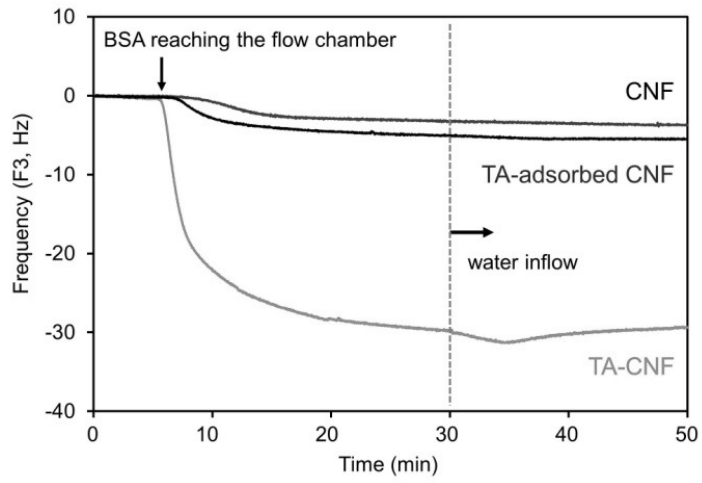

Figure 3: QCM changes in the frequency of adsorption of BSA on CNF, TA-adsorbed CNF, and TA-CNF

As shown in Figure 1a, carboxyl groups were also introduced in TA-CNF, when TA was immobilized on cellulose using BTCA. Previously, the nanofibrillation of wood powder was achieved by the introduction of carboxyl groups by esterification with maleic anhydride, which is a dicarboxylic acid. ${ }^{23}$ The electrostatic repulsion caused by the introduction of carboxyl groups on TA-CNF might be the reason suggested to contribute to the finer nanofibrillation of cellulose materials.

To evaluate the adsorption capacity of the prepared TA-CNF, the protein-adsorption property of the TA-CNF in water was evaluated by QCM analysis. QCM monitored the real-time change in mass on the surface of the nanomaterials caused by the interaction with and the adsorption of other materials. ${ }^{22,24}$ Figure 3 shows the temporal changes in the TA-CNFcoated sensor frequency when a BSA solution flowed through the chamber attached to the sensor. The negative frequency shifts indicate mass deposition in general. The decrease in the frequency of TA-CNF showed an obvious adsorption of BSA onto TA-CNF. The frequency behavior of unmodified CNF indicated that the adsorption of BSA onto the neat CNF was minor (Fig. 3). Additionally, no obvious increase in the frequency behavior after the water inflow was observed, indicating no release of non-specifically adsorbed BSA, and that BSA is specifically adsorbed on TA-CNF. QCM results indicated that a protein adsorption capacity was imparted to CNF by the immobilized TA. For comparison, the adsorption of BSA onto the TA-adsorbed CNF was also evaluated by QCM (Fig. 3). The 
frequency change showed that the BSA adsorption of TA-CNF was nearly 30 times higher than that of TA-adsorbed CNF. As shown in the ATR-FTIR results (Fig. 1b and 1c), the TA is not sufficiently retained in the CNF by simple adsorption and is desorbed relatively easily. Therefore, the QCM results suggested that the immobilization of TA on CNF contributed to the expression of the protein adsorption capacity of TA-CNF. It is also thought that the increase in the specific surface area by nanofibrillation also plays a role in improving the adsorption of BSA. In this study, we only evaluated the adsorption capacity of the surface of a TA-CNF thin film on the QCM sensor. However, since CNF can be processed into various forms, such as films, hydrogels and aerogels, ${ }^{13,14,21}$ it may be possible to prepare various support materials from TA-CNF.

\section{CONCLUSION}

Herein, we have proposed a simple, novel method for achieving the nanofibrillation of cellulose and the immobilization of specific chemical substrates on cellulose by esterification, using polycarboxylic acid. In this study, TA, which has a unique adsorption capacity, was immobilized on CNF to take advantage of the excellent properties of $\mathrm{CNF}$ as an immobilization carrier. The prepared TA-CNF had a better protein adsorption capacity than TA-adsorbed CNF. Since TA can adsorb various materials, including metallic ions, cationic dyes, and pharmacological materials, besides proteins, the use of TA-CNF may expand the application potential of $\mathrm{CNF}$ as an immobilization carrier. Further, this simple method of immobilizing specific chemical substrates on CNF can potentially be applied for preparing various types of CNFs with specific properties based on the immobilized chemical substrate.

\section{REFERENCES}

1 A. Isogai, J. Wood Sci., 59, 449 (2013), https://doi.org/10.1007/s10086-013-1365-z

2 S. Sulaiman, M. N. Mokhtar, M. N. Naim, A. S. Baharuddin and A. Sulaiman, Appl. Biochem. Biotechnol., $\quad \mathbf{1 7 5}, \quad 1817 \quad$ (2015), https://doi.org/10.1007/s12010-014-1417-x

3 M. Gopiraman, D. Deng, S. Saravanamoorthy, I.M. Chung and I. S. Kim, RSC Adv., 8, 3014 (2018), https://doi.org/10.1039/C7RA10489H

4 O. L. Galkina, V. K. Ivanov, A. V. Agafonov, G. A. Seisenbaeva and V. G. Kessler, J. Mater. Chem. B, 3, 1688 (2015), https://doi.org/10.1039/C4TB01823K
5 H. A. M. Bacelo, S. C. R. Santos and C. M. S. Botelho, Chem. Eng. J., 303, 575 (2016), https://doi.org/10.1016/j.cej.2016.06.044

6 Z. Gao and I. Zharov, Chem. Mater., 26, 2030 (2014), https://doi.org/10.1021/cm4039945

7 A. Dutta and S. K. Dolui, Appl. Surf. Sci., 257, 6889

(2011),

https://doi.org/10.1016/j.apsusc.2011.03.025

8 Y. Ju, J. C. Cui, M. Müllner, T. Suma, M. Hu et al., Biomacromolecules, 16, $807 \quad$ (2015), https://doi.org/10.1021/bm5017139

9 H. Park, K. Kim, J. Lee, J. Y. Choi, D. Hong et al., Angew. Chem. Int. Ed., 53, 12420 (2014), https://doi.org/10.1002/anie.201405905

10 P. Thitiwongsawet, N. Wichapeng and A. Waiyaroj, Adv. Mater. Res., 774, $896 \quad$ (2013), https://doi.org/10.4028/www.scientific.net/AMR.774776.896

11 X. Huang, Y. P. Wang, X. P. Liao and B. Shi, J. Hazard. Mater., 183, $793 \quad$ (2010), https://doi.org/10.1016/j.jhazmat.2010.07.096

12 I. Chibata, T. Tosa, T. Mori, T. Watanabe and N. Sakata, Enzyme Microb. Tech., 8, 130 (1986), https://doi.org/10.1016/0141-0229(86)90099-2

13 B. Zhou, X. Hu, J. Zhu, Z. Wang, X. Wang et al., Int. J. Biol. Macromol., 91, 68 (2016), https://doi.org/10.1016/j.ijbiomac.2016.05.084

14 Y. Pei, S. Chu, Y. Chen, Z. Li, J. Zhao et al., Int. J. Biol. Macromol., 103, $254 \quad$ (2017) https://doi.org/10.1016/j.ijbiomac.2017.05.072

15 C. M. Welch, Text. Res. J., 58, 480 (1988), https://doi.org/10.1177\%2F004051758805800809

16 B. Ji, K. Yan and G. Sun, Ind. Eng. Chem. Res., 55, 5216 (2016), https://doi.org/10.1021/acs.iecr.6b00159

17 T. Saito, Y. Nishiyama, J.-L. Putaux, M. Vignon and A. Isogai, Biomacromolecules, 7, 1687 (2006), https://doi.org/10.1021/bm060154s

18 Y. Noguchi, I. Homma and Y. Matsubara, Cellulose, 24, 1295 (2017), https://doi.org/10.1007/s10570-0171191-3

19 J. A. Sirviö, J. Ukkola and H. Liimatainen, Cellulose, 26, $2303 \quad$ (2019), https://doi.org/10.1007/s10570-019-02257-8

20 W. Ge, S. Cao, F. Shen, Y. Wang, J. Ren et al., Carbohyd. Polym., 224, $115147 \quad$ (2019) https://doi.org/10.1016/j.carbpol.2019.115147

${ }_{21}$ Y. Ji, Y. Wen, Z. Wang, S. Zhang and M. Guo, J. Clean. Prod., 255, $120276 \quad$ (2020), https://doi.org/10.1016/j.jclepro.2020.120276

22 A. Kumagai, S.-H. Lee and T. Endo, Biomacromolecules, 14, $2420 \quad$ (2013), https://doi.org/10.1021/bm400553s

23 S. Iwamoto and T. Endo, ACS Macro Lett., 4, 80 (2015), https://doi.org/10.1021/mz500787p

24 A. Dolatshahi-Pirouz, K. Rechendorff, M. B. Hovgaard, M. Foss, J. Chevallier et al., Colloid. $\begin{array}{llll}\text { Surface. } & B, & 66 & 53\end{array}$ https://doi.org/10.1016/j.colsurfb.2008.05.010 